\title{
Network Pharmacology-Based Prediction of the Active Ingredients and Potential Targets of Chinese Herbal Danyu Gukang Pills for Application to Osteonecrosis of the Femoral Head Disease
}

\section{Yongchang Guo}

Zhengzhou Traditional Chinese Hospital of Orthopaedics

\section{Dapeng Zhang}

Zhengzhou Traditional Chinese Hospital of Orthopaedics

Yuju Cao ( $\nabla$ y1972783422@163.com )

Zhenzhou Tradtional Chinese Hospital of Orthopaedics

Xiaoyan Feng

Zhengzhou Traditional Chinese Hospital of Orthopaedics

\section{Caihong Shen}

Zhengzhou Traditional Chinese Hospital of Orthopaedics

Wenqian Li

Zhengzhou Traditional Chinese Hospital of Orthopaedics

\section{Shunguo Gong}

Zhengzhou Traditional Chinese Hospital of Orthopaedics

\section{Fengzhi Hou}

Zhengzhou Traditional Chinese Hospital of Orthopaedics

\section{Zhimin Yang}

Zhengzhou Traditional Chinese Hospital of Orthopaedics

Juanjuan Yi

Zhengzhou Traditional Chinese hospital of Othopaedics

\section{Dan Luo}

Zhengzhou Traditional Chinese Hospital of Orthopaedics

\section{Xifeng Chen}

Zhengzhou Traditional Chinese Hospital of Othopaedics Jingbo Song

Zhengzhou Treditional Chinese Hospital of Orthopaedics

\section{Research}


Keywords: Danyu Gukang Pill, Osteonecrosis of the femoral head, network pharmacology

Posted Date: January 13th, 2021

DOl: https://doi.org/10.21203/rs.3.rs-143355/v1

License: (c) (1) This work is licensed under a Creative Commons Attribution 4.0 International License. Read Full License 


\section{Abstract}

Ethnopharmacological relevance

Osteonecrosis of the femoral head (ONFH) is still a challenge for orthopedists worldwide, which may lead to disability in patients without effective treatment. A newly developed formula of Chinese medicine, Danyu Gukang Pills (DGP), was recognized to be effective for ONFH. Nevertheless, its molecular mechanisms remain to be clarified.

\section{Methods}

Network pharmacology was adopted to detect the mechanism of DGP on ONFH. The compounds of DGP were collected from the online databases, and active components were selected based on their $\mathrm{OB}$ and DL index. The potential proteins of DGP were acquired from TCMSP database, while the potential genes of ONFH were obtained from Gene Cards and Pubmed Gene databases. The function of Gene and potential pathways were researched by GO and KEGG pathway enrichment analysis. The compoundstargets and targets-pathways network were constructed in an R and Cytosacpe software. The mechanism was further investigated via molecular docking. Finally, in-vitro experiments were validated in the BMSCs.

Results

A total of 2305 compounds in DGP were gained, among which, 370 were selected as active components for which conforming to criteria. Combined the network analysis, molecular docking and in-vitro experiments, the results firstly demonstrated that the treatment effect of DGP on ONFH may be closely related to HIF-1a, VEGFA and HIF-1 signaling pathway.

Conclusion

The current study firstly researched the molecular mechanism of DGP on ONFH based on network pharmacology. The results indicated that DGP may exert the effect on ONFH targeting on HIF-1a and VEGFA via HIF-1 signaling pathway.

\section{Background}

Osteonecrosis of the femoral head (ONFH) is also defined as avascular necrosis or aseptic necrosis, which is characterized by impaired blood flow to the bone due to trauma or non-traumatic blood flow, resulting in bone cell death[1,2]. The pathogenic factors of ONFH are complex so that it is difficult to diagnosis at the early stage. It has been reported that about $80 \%$ of patients will suffer from femoral head collapse in 1 to 4 years and finally leading to necrosis even disability without effective treatment [3]. Studies indicated that in China, there were about 8.12 million cases of ONFH in cohort over the age of 15 years; And there were about 11.76 cases of ONFH per 100,000 people in the general rural population, while 9.57 ONFH patients per 100,000 people in urban population[4, 5]. Importantly, due to the high rate of disability and increasing incidence of ONFH among population aged from 30 to 50 , the patients might 
lose the ability to work, which undoubtedly causes a huge burden to the patients' family and even the society.

Up to now, there is still a lack of a simple and effective treatment for ONFH, therefore ONFH has always been regarded as a major problem in the field of orthopedics. With the development of ONFH to the late stage, patients will have to carry out total hip arthroplasty (THA), however, the trauma and economic pressure associated with the surgery will also become a burden for patients[6, 7]. Therefore, it is crucial to research the methods of retain the femoral head of patients, especially the young patients. In conservative treatment, bisphosphonates, statins and anticoagulants were medicine which commonly use[8, 9]. Although the medicine mentioned above can partially restore the function of bone cells through different pharmacological mechanisms, the obvious side effects such as gastrointestinal stimulation, renal toxicity or mandibular joint necrosis cannot be ignored [10]. Complementary medicine and alternative medicine (CAM) are commonly used for the treatment of ONFH due to the absence of specific agents. In China, traditional Chinese medicine (TCM) has been used for hundreds of years to treat ONFH which named bone erosion in Huang Di Nei Jing. Zhang et,al performed a systematic review and metaanalysis of randomized controlled trials, their results indicated that TCM take essential part in the adjuvant treatment for ONFH [11].

Danyu Gukang Pills (DGP) as a new method in the treatment of ONFH developed by our research group, is possible to cure patients with ONFH in the early stage. This formular was consist mainly of the herb medicine which function as promoting blood circulation and removing blood stasis, such as Sanqi, Dahuang, Ruxiang, Moyao, Yanhusuo, Danshen; And Chuanxiong, Muxiang, Yujin to relieve pain. Since the development of DGP, it was considered as an effective way to treat the ONFH. However, its mechanism of action is still unclear, which may be related to the drug-target interaction network of "multicomponent, multi-target".

From the view of chemistry, TCM formula is a complicate system with multiple targets as well as interactions among their substances [12]. Different from western medicine of "one target, one drug", TCM emphasized the concept of the whole human body. Network pharmacology combines the systems biology with pharmacokinetic and pharmacodynamics to research medicines, targets and their pharmacological activities [13]. Systematically, network pharmacology is on the basis of the interactions of disease-gene-target-drug networks to observe the effects of drugs on diseases [14, 15]. This research methods is in accordance with the theory that TCM emphasizes the diagnosis and treatment of diseases from a holistic perspective, and the synergistic effect of TCM and its compounds $[16,17]$.

In the current study, we used the approaches of network pharmacology and molecular docking to investigate the potential molecular mechanisms of DGP on ONFH the interactions between activated compounds and protein targets. Additionally, in vitro experiments on human bone marrow mesenchymal stem cells (BMSCs) which played crucial role in the process of osteogenesis were also conducted to validate the potential underlying mechanism of DGP on ONFH. The detailed technical strategy of the current study was shown in Figure 1. 


\section{Materials And Methods}

All compounds of the 19 Chinese medicine herbs in DGP were obtained from the traditional Chinese medicine systems pharmacology (TCMSP: http://tcmspw.com/)[18] and Bioinformatics analysis tool for molecular mechanism of Traditional Chinese Medicine (BATMAN-TCM: http://bionet.ncpsb.org/batmantcm/index.php) [19] databases.

\section{Searching Strategy for Bioactive Compounds in DGP}

To be effective, oral TCM must overcome the obstacles in the process of absorption, distribution, metabolism and excretion (ADME). Oral bioavailability (OB) is one of the most important pharmacokinetic parameters in ADME. High $\mathrm{OB}$ is commonly necessary to determine the drug-likeness $(\mathrm{DL})$ index of bioactive substances. The components with $\mathrm{OB} \geq 30 \%$ were considered to have high $\mathrm{OB}$.

The DL index is crucial for rapid screening of active compounds as a qualitative concept when it was applied in drug design to estimate the druggability of a molecule. In the DrugBank database, the components with $\mathrm{DL} \geq 0.18$ were considered to have high druggability. Therefore, the components in DGP with $O B \geq 30 \%$ and $D L \geq 0.18$ were selected as active substances in our study.

\section{Prediction of Herbs' Targets for DGP}

The protein targets of the active compounds in DGP were obtained from the TCMSP databases (http://tcmspw.com/).

\section{Collection of Gene Targets for ONFH}

ONFH-related genes of homo-sapiens were collected from two databases, Pubmed Gene (https://www.ncbi.nlm.nih.gov/gene/) and Gene Cards (https://www.genecards.org).

\section{GO and KEGG Pathway Enrichment Analysis for ONFH-Related Targets of DGP}

The gene ontology (GO) and Kyoto Encyclopedia of Genes and Genomes (KEGG) pathway enrichment analyses were conducted using the R Project for Statistical Computing. (Version 3.6.3)

\section{Interaction Network Construction and Analysis}

To investigate the in-depth molecular mechanism of DGP on ONFH, the compounds-targets and targetspathways networks were constructed by Cytoscape software (Version. 3.6.1). In these networks, the components, proteins, or pathways were represented as nodes, whereas the interactions between nodes were represented as edges.

\section{Molecular Docking}

In order to study the association between quercetin and its crucial targets in the network, molecular docking approach was applied to analyzed the strength and mode of interactions between quercetin and 
HIF-1a or VEGFA. The protein crystal structure of HIF-1a or VEGFA were obtained from Protein Data Bank (PDB ID: 4H6J for HIF-1a and 5DN2 for VEGFA). The process is simply described as follows: ChemDraw and Chem3D were applied to prepare the chemical structure of quercetin. Autodock vina software (version: 1.1.2)[20] was applied for molecular docking. Discovery Studio was used to analyze the results of docking and presented as final figures.

\section{Experimental Validation}

\section{Preparation of DGP Aqueous Solution}

DGP was developed and manufactured by our research group. It contains the following herbs: Danshen, Yujin, Sanqi, Jixueteng, Niuxi, Xuduan, Gusuibu, Gouqi, Danggui, Chuanxiong, Shudihuang, Dangshen, Baizhu,Ruxiang, Moyao, Yanhusuo, Muxiang and Wujiapi. $0.2 \mathrm{~g}$ DGP was dissolved in $40 \mathrm{ml}$ double steaming water to make a $5 \mathrm{mg} / \mathrm{ml}$ stock solution which was then added to the medium at the indicated concentrations.

\section{Cell culture}

Human bone marrow mesenchymal stem cells (BMSCs) were commercially obtained from Procell Life Science \& Technology Co., Ltd (Cat No.: CP-H166, Wuhan, China). BMSCs were cultured in MEM-a medium with $10 \%$ FBS, $100 \mathrm{U} / \mathrm{ml}$ penicillin, and $100 \mathrm{mg} / \mathrm{ml}$ streptomycin. Routinely, cells were maintained at $37^{\circ} \mathrm{C}$ with $5 \% \mathrm{CO}_{2}$.

\section{Cell Counting Kit-8 (CCK8) Assay}

Adjust the density of the cells in the control and the DP groups to be tested to $5 \times 10^{3}$, and inoculated in 96-well plates. Cell proliferation was assessed by CCK8 method according manufacturer's instructions. CCK8 (final concentration $5 \mathrm{mg} / \mathrm{ml}$ ) was added to each well incubated at $37^{\circ} \mathrm{C} 4 \mathrm{~h}$, discarding the supernatant and adding DMSO. Absorbance value (OD) at A570 $\mathrm{nm}$ was measured using a microplate reader. The experimental results were repeated three times independently.

\section{Quantitative Real-Time PCR}

Total RNA of cells was extracted using TRIzol reagent (Invitrogen, Carlsbad, CA, USA) according to the manufacturer's instructions. After detecting the concentration, we synthesized cDNA of the genes by using the One Step PrimeScript cDNA Synthesis Kit (Takara Biotechnology, Dalian, China). After reverse transcription, real-time PCR was performed using the Applied Biosystems 7500 Sequence Detection system (Applied Biosystems, Foster City, CA, USA). The HIF-1a and VEGFA expression were normalized to GAPDH (HIF-1 a forward 5'-GCCTCTGTGATGAGGCTTACC-3' and reverse 5'-

CAGTGCAATACCTTCCATGTTGC-3'; VEGFA forward 5'-ACTGCCATCCAATCGAGACC-3' and reverse 5'TTGATCCGCATAATCTGCATGGT-3'; GAPDH forward 5'- GAGTCAACGGATTTGGTCGT -3' and reverse 5'GACAAGCTTCCCGTTCTCAG -3'). Relative results were calculated using the $2-\triangle \triangle C T$ equation. 


\section{Data analysis}

Data are presented as mean \pm standard deviation (SD). Statistical differences were analyzed with SPSS version 20.0 (SPSS Inc., Chicago, IL, USA) using one-way ANOVA followed by Bonferroni post hoc tests.

\section{Results}

\section{Identification of Bioactive Substances in DGP}

2305 components in DGP were obtained from TCMSP and BATMAN-TCM databases, the remaining components were 370 after threshold screening ( $O B \geq 30 \%$ and $D L \geq 0.18)$ (Table 1).

\section{Target identification of DGP on ONFH}

For the 370 candidate bio-active substances, 4990 protein targets were collected from TCMSP databases. And then, 1413 protein targets were remained for the next analyses after eliminating the overlaps. 124 ONFH-related gene of homo-sapiens were collected from Pubmed Gene and Gene card databases. As shown in Figure 2, all herb protein targets for herb-related and ONFH-related proteins were listed as two separate sets. A Venn diagram is obtained by using the closed loop form of fixed position to represent the set and its relations. As results, 18 targets of 44 components in DGP were associated with ONFH. The information of 18 targets and the interaction degrees between the targets were shown in Table 2.

\section{Compounds-Targets Network and Analysis}

Traditional Chinese medicine formulas were characteristic for their multi-target and multipharmacological effects. It is of great significance to research the internal mechanism of Chinese medicine formulas in the treatment of complicate diseases through the analysis of networks. In our current study, we contrasted the components-targets network of DGP on ONFH by R/Bioconductor platform (Figure 3), which included 62 nodes (44 for bio-active components and 18 for gene targets). Among these compounds, there were several high-degree substances associated with multiple ONFH targets, mostly including quercetin (MOL000098), luteolin (MOL000006). These targets in the network were potentially represent the essential therapeutic effects of DGP on ONFH.

\section{Gene Functions and Pathway Enrichment Prediction}

In order to clarify the biological characteristics of the potential targets of DGP on ONFH, Gene Ontology (GO) and Kyoto Encyclopedia of Genes and Genomes (KEGG) pathway enrichment analysis were performed by R/Bio-conductor platform. As shown in Figure 4, there were listed the top 20 (Count $\geq 2$ and $p<0.05)$ significantly enriched terms in functions of these targets, which revealed that DGP may regulate the development of ONFH via cytokine activity, receptor ligand activity, growth factor activity, cytokine receptor linking to their treatment effect on ONFH. The involved pathways of DGP on ONFH were detected using KEGG analysis. As results, the significant pathways of DGP on ONFH were shown in Figure 5. 


\section{Targets-Pathways Network Analysis}

In order to further clarify the molecular mechanism by which DGP alleviates ONFH, we constructed a targets-pathways network according to the relevant proteins and their relevant signaling pathways. As shown in Figure 6, the network included 33 nodes (13 for proteins and 20 for pathways). From these potential pathways, Proteoglycans in cancer, AGE-RAGE signaling pathway in diabetic complications, HIF1 signaling pathway have been the top three degrees value. However, Proteoglycans in cancer and AGERAGE signaling pathway in diabetic complications were closely related to the cancer and diabetic complications respectively. HIF-1 signaling pathway was most associated with the effect of DGP on ONFH. Among these potential targets, VEGF, TP53 and TGFB1 were recognized as relatively high-degree targets, which played essential role in development of ONFH. From the combined drug target prediction, GO analysis, KEGG pathway enrichment analysis as well as targets-pathway network, we found that the pharmacology effects of DGP on ONFH might to be the regulation of VEGFA through HIF-1 signaling pathway. The detailed of HIF-1 signaling pathway was shown in Figure 7.

\section{Molecular Docking Analysis}

To investigate the reliability of molecular-target interaction and research accurate binding patterns, we chose quercetin as crucial molecular and HIF-1a or VEGFA as key targets according to the results of network analysis above. The results indicated that there was stronger interaction between quercetin and HIF-1a or VEGFA. As shown in Figure 8A, quercetin combined to HIF-1a mainly interacted with amino acid residues of SER by conventional hydrogen bond, TYR by pi-pi stacked and pi-donor hydrogen bond, ARG by unfavorable donor-donor. And in Figure 8B, quercetin combined to VEGFA mainly interacted with amino acid resides of THR, ILE, HIS by conventional hydrogen bond, TYR by conventional hydrogen bond and pi-pi stacked or pi-pi T-shaped, ASP by pi-anion.

\section{Experimental Validation}

\section{DGP could improve the viability of BMSCs}

The viability of BMSCs increased significantly after DGP treating for $24 \mathrm{H}$ (Figure 9A). By using CCK-8 assay, the results indicated that $0.1,0.25$ and $0.5 \mathrm{mg} / \mathrm{ml} \mathrm{DGP}$ treatment for $12,24,36$ and $48 \mathrm{H}$ could promote the proliferation of BMSCs compared with control group. However, $0.25 \mathrm{mg} / \mathrm{ml}$ DGP treatment obtained the best efficacy than the other two (Figure 9B).

\section{DGP improve the mRNA expression of HIF-1a and VEGFA}

In BMSCs, the consequence of the RT-PCR test demonstrated that 0.1 and $0.25 \mathrm{mg} / \mathrm{ml} \mathrm{DGP}$ treating could significantly increase the expression of HIF-1a and VEGFA mRNA, while $0.5 \mathrm{mg} / \mathrm{ml} \mathrm{DGP} \mathrm{treating} \mathrm{had} \mathrm{a}$ reverse effect (Figure 10).

\section{Discussion}


Femoral head necrosis (ONFH) is a refractory disease characterized by damaged subchondral microcirculation, skeletal necrosis and microfracture accumulation without continuous remodeling[21]. The pathological mechanism of ONFH is very complicate, which are associated with multiple proteins and pathways during the development and procession[22]. TCM is commonly composed of a variety of compounds, with a wide range of pharmacological activities and a variety of targets and pathways[23]. Accumulating evidence indicated that TCM may benefit the treatment of ONFH[11, 24, 25]. Nevertheless, this characteristic of TCM may make it difficult to further study of the underlying mechanisms. Network pharmacology is an organic combination of system biology and omics, which can provide a direction for the mechanism research of complex TCM [26]. In this research, we used these methods to elucidate the pharmacological mechanisms by which DGP alleviates ONFH.

Among the TCM, components lacking appropriate pharmacokinetic properties cannot arrive to the target organ and then transmit biological activity. Thus, we screen the active compounds in the DGP with OB $\geq$ $30 \%, \mathrm{DL} \geq 0.18$, which are considered pharmacokinetic active[18]. Importantly, it has been realized that components with high-degree may represent the crucial treating effect of DGP on ONFH. In our research, quercetin was the most significant components, and then, the luteolin, sesamin and kaempferol were followed. Qucercetin, a natural flavonoid, was confirmed to promote BMSCs proliferation and osteogenic differentiation[27, 28]. BMSCs have powerful self-proliferation ability and multi-potential differentiation capacity and can undergo osteogenesis through induction. Recently, BMSCs have been researched and a new technology has been developed and applied for regenerative medicine. Numerous reports have indicated successful results of the treatment of ONFH by using stem cell transplantation. [29-31].

According to the results above, DGP may exert its pharmacological functions on the treatment of ONFH through regulation of cytokine activity and growth factor activity, which was considered as the important mechanism of ONFH progression[32, 33]. As predicted by network pharmacology, DGP may play therapeutic role on ONFH mainly through hypoxia-inducible factor-1 (HIF-1) signaling pathway and targeting to the HIF-1a and vascular endothelial growth factor A (VEGFA). The transcription factor HIF-1 is a key regulatory factor responsible for the induction of genes that promote cell adaptation and survival and the entire organism from normal oxygen to hypoxia. HIF-1a is a subtype discovered by the identification of a hypoxia response element. The functional HIF-1 is composed of two subunits, HIF-1a and HIF-1 $\beta$, with the HIF-1 a subunit being responsive to hypoxia[34, 35]. It has been reported that HIF-1a can improve the expression of RUNX-2, OCN, and ALP in BMSCs[36]. Meanwhile, the over-expression of HIF-1a can promote the differentiation from BMSCs to osteoblasts after the osteogenesis induction[37]. Moreover, VEGF is regulated by HIF-1a. The over-expression of HIF-1a enhanced the secretion of VEGF in $\operatorname{BMSCs}[38,39]$. VEGF is the currently recognized as the growth factor with the strongest ability to promote angiogenesis, which could improve the proliferation and differentiation of endothelial cells and induce the formation of new vessels[40].

To further validate the prediction of the targets of DGP, we used molecular docking. The results demonstrated that both HIF-1 $a$ and VEGFA had a high interaction with quercetin by combined with amino acid residues. Furthermore, we designed an in vitro experiment to validate the postulation, which DGP 
may alleviated ONFH via HIF-1 signaling pathway. The results showed that DGP treatment significantly increased BMSCs viability and RT-PCR assay indicated that DGP treatment could improve the mRNA expression of HIF-1 $a$ and VEGFA. These results revealed that DGP might exert the effect on ONFH though HIF-1 signaling pathway. The in-depth molecular mechanism needs further clarified.

\section{Conclusion}

To summary, our current research firstly gives a systematic view of potential targets and pathways relevant to the treating effect of DGP on ONFH. Importantly, for the further confirmation, we still need more in-depth molecular experiments to verify the predication from the network pharmacology.

\section{Abbreviations}

BATMAN-TCM, Bioinformatics Analysis Tool for Molecular Mechanism of Traditional Chinese Medicine BMSCs, bone marrow mesenchymal stem cells

DL, Drug-likeness

DGP, Danyu Gukang Pill

GO, Gene ontology

HIF-1a, Hypoxia-inducible factor-1 alpha

KEGG, Kyoto Encyclopedia of Genes and Genomes

$\mathrm{OB}$, Oral bioavailability

ONFH, Osteonecrosis of the Femoral Head

TCM, Traditional Chinese Medicine

TCMSP, Traditional Chinese Medicine Systems Pharmacology

VEGFA, vascular endothelial growth factor $A$

\section{Declarations}

\section{Ethics approval and consent to participate}

All compounds of the 19 Chinese medicine herbs in DGP were obtained from the traditional Chinese medicine systems pharmacology (TCMSP: http://tcmspw.com/)[18] and Bioinformatics analysis tool for molecular mechanism of Traditional Chinese Medicine (BATMAN-TCM: http://bionet.ncpsb.org/batman- 
tcm/index.php) [19] databases.All participants signed informed consent forms before participating in this study.

\section{Consent for publication}

Not applicable.

\section{Availability of data and materials}

All data generated or analysed during this study are included in this published article.

\section{Competing interests}

The authors declare that they have no competing interests

\section{Funding}

TCM Scientific Research Subject of Chinese Medicine Administration Bureau of Henan (2018ZY3011).

\section{Authors' Contributions}

Y C, D Z and X F conceived and designed the project.

C S, W L and S G recruited and collected study samples.

$\mathrm{F} \mathrm{H}, \mathrm{Z} \mathrm{Y}$ and $\mathrm{J} \mathrm{Y}$ selected the SNPs and designed primers.

Y G, Y C, X F and C S performed the experiments.

$D L, X C$ and $J$ S performed and analyzed the data.

Y G and D Z wrote and revised the manuscript.

\section{Acknowledgments}

We would like to thank all the TCM research project funds of Henan administration of traditional Chinese medicine for their support.

\section{References}

[1] Cohen-Rosenblum A, Cui Q. Osteonecrosis of the Femoral Head. Orthop Clin North Am, 2019; 50: 139149.

[2] Petek D, Hannouche D, Suva D. Osteonecrosis of the femoral head: pathophysiology and current concepts of treatment. EFORT Open Rev, 2019; 4: 85-97. 
[3] Papakostidis C, Tosounidis TH, Jones E, Giannoudis PV. The role of "cell therapy" in osteonecrosis of the femoral head. A systematic review of the literature and meta-analysis of 7 studies. Acta Orthop, 2016; 87: 72-8.

[4] Liu LH, Zhang QY, Sun W, Li ZR, Gao FQ. Corticosteroid-induced Osteonecrosis of the Femoral Head: Detection, Diagnosis, and Treatment in Earlier Stages. Chin Med J (Engl), 2017; 130: 2601-2607.

[5] Chinese Guideline for the Diagnosis and Treatment of Osteonecrosis of the Femoral Head in Adults. Orthop Surg, 2017; 9: 3-12.

[6] Issa K, Pivec R, Kapadia BH, Banerjee S, Mont MA. Osteonecrosis of the femoral head: the total hip replacement solution. Bone Joint J, 2013; 95-b: 46-50.

[7] Gao YS, Ai ZS, Zhu ZH, Yu XW, Zhang CQ. Injury-to-surgery interval does not affect postfracture osteonecrosis of the femoral head in young adults: a systematic review. Eur J Orthop Surg Traumatol, 2013; 23: 203-9.

[8] Guideline for Diagnostic and Treatment of Osteonecrosis of the Femoral Head. Orthop Surg, 2015; 7: 200-7.

[9] Larson E, Jones LC, Goodman SB, Koo KH, Cui Q. Early-stage osteonecrosis of the femoral head: where are we and where are we going in year 2018? Int Orthop, 2018; 42: 1723-1728.

[10] Liu J, Zhang Z, Guo Q, Dong Y, Zhao Q, Ma X. Syringin prevents bone loss in ovariectomized mice via TRAF6 mediated inhibition of NF-kappaB and stimulation of PI3K/AKT. Phytomedicine, 2018; 42: 43-50.

[11] Zhang Q, Yang F, Chen Y, Wang H, Chen D, He W, Chen P. Chinese herbal medicine formulas as adjuvant therapy for osteonecrosis of the femoral head: A systematic review and meta-analysis of randomized controlled trials. Medicine (Baltimore), 2018; 97: e12196.

[12] Li S, Zhang B. Traditional Chinese medicine network pharmacology: theory, methodology and application. Chin J Nat Med, 2013; 11: 110-20.

[13] Ning K, Zhao X, Poetsch A, Chen WH, Yang J. Computational Molecular Networks and Network Pharmacology. Biomed Res Int, 2017; 2017: 7573904.

[14] Ye H, Wei J, Tang K, Feuers R, Hong H. Drug Repositioning Through Network Pharmacology. Curr Top Med Chem, 2016; 16: 3646-3656.

[15] Zhang W, Bai Y, Wang Y, Xiao W. Polypharmacology in Drug Discovery: A Review from Systems Pharmacology Perspective. Curr Pharm Des, 2016; 22: 3171-81.

[16] Zhang R, Zhu X, Bai H, Ning K. Network Pharmacology Databases for Traditional Chinese Medicine: Review and Assessment. Front Pharmacol, 2019; 10: 123. 
[17] Wu CW, Lu L, Liang SW, Chen C, Wang SM. [Application of drug-target prediction technology in network pharmacology of traditional Chinese medicine]. Zhongguo Zhong Yao Za Zhi, 2016; 41: 377-382.

[18] Ru J, Li P, Wang J, Zhou W, Li B, Huang C, Li P, Guo Z, Tao W, Yang Y, Xu X, Li Y, Wang Y, Yang L. TCMSP: a database of systems pharmacology for drug discovery from herbal medicines. J Cheminform, 2014; 6: 13.

[19] Liu Z, Guo F, Wang Y, Li C, Zhang X, Li H, Diao L, Gu J, Wang W, Li D, He F. BATMAN-TCM: a Bioinformatics Analysis Tool for Molecular mechANism of Traditional Chinese Medicine. Sci Rep, 2016; 6: 21146.

[20] Trott O, Olson AJ. AutoDock Vina: improving the speed and accuracy of docking with a new scoring function, efficient optimization, and multithreading. J Comput Chem, 2010; 31: 455-61.

[21] Moya-Angeler J, Gianakos AL, Villa JC, Ni A, Lane JM. Current concepts on osteonecrosis of the femoral head. World J Orthop, 2015; 6: 590-601.

[22] Baig SA, Baig MN. Osteonecrosis of the Femoral Head: Etiology, Investigations, and Management. Cureus, 2018; 10: e3171.

[23] Liu ZH, Sun XB. [Network pharmacology: new opportunity for the modernization of traditional Chinese medicine]. Yao Xue Xue Bao, 2012; 47: 696-703.

[24] Yu T, Zhang Z, Xie L, Ke X, Liu Y. The influence of traditional Chinese medicine constitutions on the potential repair capacity after osteonecrosis of the femoral head. Complement Ther Med, 2016; 29: 89-93.

[25] Yeh YA, Chiang JH, Wu MY, Tsai CH, Hsu HC, Hsu HC, Lin TL. Association of Traditional Chinese Medicine Therapy with Risk of Total Hip Replacement in Patients with Nontraumatic Osteonecrosis of the Femoral Head: A Population-Based Cohort Study. Evid Based Complement Alternat Med, 2019; 2019: 5870179.

[26] Boezio B, Audouze K, Ducrot P, Taboureau O. Network-based Approaches in Pharmacology. Mol Inform, 2017; 36.

[27] Pang XG, Cong Y, Bao NR, Li YG, Zhao JN. Quercetin Stimulates Bone Marrow Mesenchymal Stem Cell Differentiation through an Estrogen Receptor-Mediated Pathway. Biomed Res Int, 2018; 2018 : 4178021.

[28] Zhou Y, Wu Y, Jiang X, Zhang X, Xia L, Lin K, Xu Y. The Effect of Quercetin on the Osteogenesic Differentiation and Angiogenic Factor Expression of Bone Marrow-Derived Mesenchymal Stem Cells. PLoS One, 2015; 10: e0129605.

[29] Wang C, Wang Y, Meng HY, Yuan XL, Xu XL, Wang AY, Guo QY, Peng J, Lu SB. Application of bone marrow mesenchymal stem cells to the treatment of osteonecrosis of the femoral head. Int $\mathrm{J}$ Clin Exp 
Med, 2015; 8: 3127-35.

[30] Li R, Lin QX, Liang XZ, Liu GB, Tang H, Wang Y, Lu SB, Peng J. Stem cell therapy for treating osteonecrosis of the femoral head: From clinical applications to related basic research. Stem Cell Res Ther, 2018; 9: 291.

[31] Zhao D, Liu Y, Ma C, Gu G, Han DF. A Mini Review: Stem Cell Therapy for Osteonecrosis of the Femoral Head and Pharmacological Aspects. Curr Pharm Des, 2019; 25: 1099-1104.

[32] Kuroda Y, Kawai T, Goto K, Matsuda S. Clinical application of injectable growth factor for bone regeneration: a systematic review. Inflamm Regen, 2019; 39: 20.

[33] Zou D, Zhang K, Yang Y, Ren Y, Zhang L, Xiao X, Zhang H, Liu S, Li J. Th17 and IL-17 exhibit higher levels in osteonecrosis of the femoral head and have a positive correlation with severity of pain. Endokrynol Pol, 2018; 69: 283-290.

[34] Hong JM, Kim TH, Chae SC, Koo KH, Lee YJ, Park EK, Choi JY, Ryoo HM, Kim SY. Association study of hypoxia inducible factor 1alpha (HIF1alpha) with osteonecrosis of femoral head in a Korean population. Osteoarthritis Cartilage, 2007; 15: 688-94.

[35] Ma W, Xin K, Chen K, Tang H, Chen H, Zhi L, Liu H. Relationship of common variants in VEGFA gene with osteonecrosis of the femoral head: A Han Chinese population based association study. Sci Rep, 2018; 8: 16221.

[36] Shao J, Zhang Y, Yang T, Qi J, Zhang L, Deng L. HIF-1alpha disturbs osteoblasts and osteoclasts coupling in bone remodeling by up-regulating OPG expression. In Vitro Cell Dev Biol Anim, 2015; 51: 80814.

[37] Stegen S, van Gastel N, Eelen G, Ghesquiere B, D'Anna F, Thienpont B, Goveia J, Torrekens S, Van Looveren R, Luyten FP, Maxwell PH, Wielockx B, Lambrechts D, Fendt SM, Carmeliet P, Carmeliet G. HIF1alpha Promotes Glutamine-Mediated Redox Homeostasis and Glycogen-Dependent Bioenergetics to Support Postimplantation Bone Cell Survival. Cell Metab, 2016; 23: 265-79.

[38] Yu J, Liang F, Huang H, Pirttiniemi P, Yu D. Effects of loading on chondrocyte hypoxia, HIF-1alpha and VEGF in the mandibular condylar cartilage of young rats. Orthod Craniofac Res, 2018; 21: 41-47.

[39] Xu J, Sun Y, Wu T, Liu Y, Shi L, Zhang J, Kang Q, Chai Y, Li G. Enhancement of bone regeneration with the accordion technique via HIF-1alpha/VEGF activation in a rat distraction osteogenesis model. $\mathrm{J}$ Tissue Eng Regen Med, 2018; 12: e1268-e1276.

[40] Hu K, Olsen BR. Osteoblast-derived VEGF regulates osteoblast differentiation and bone formation during bone repair. J Clin Invest, 2016; 126: 509-26. 


\section{Tables}

Table 1 Number of components in DGP with $\mathrm{OB} \geq 30 \%$ and $\mathrm{DL} \geq 0.18$

\begin{tabular}{|llll|}
\hline Herbs & Latin name & Total & OB $\geq 30 \%$ \\
Danshen & Radix Salviae & 202 & 65 \\
Yujin & Curcumae Radix $\geq 0.18$ \\
\hline Sanqi & Panax Notoginseng & 222 & 15 \\
\hline Jixueteng & Spatholobus Suberectus Dunn & 119 & 8 \\
\hline Niuxi & Achyranthis Bidentatae Radix & 176 & 24 \\
\hline Xuduan & Dipsaci Radix & 31 & 8 \\
\hline Gusuibu & Drynariae Rhizoma & 71 & 18 \\
\hline Gouqizi & Lycii Fructus & 188 & 45 \\
\hline Danggui & Angelicae Sinensis Radix & 125 & 2 \\
\hline Chuanxiong & Chuanxiong Rhizoma & 189 & 7 \\
\hline Shudihuang & Rehmanniae Radix Praeparata & 76 & 2 \\
\hline Dangshen & Codonopsis Radix & 134 & 21 \\
\hline Baizhu & Atractylodes Macrocephala Koidz & 55 & 7 \\
\hline Ruxiang & Olibanun & 127 & 8 \\
\hline Moyao & Myrrha & 276 & 45 \\
\hline Yanhusuo & Corydalis Rhizoma & 77 & 49 \\
\hline Dahuang & Radix Rhei Et Rhizome & 106 & 6 \\
\hline Muxiang & Aucklandiae Radix & 16 & 4 \\
\hline Wujiapi & Acanthopanax gracilistylus & & 24 \\
\hline
\end{tabular}

Table 2 Targets of DGP on ONFH 


\begin{tabular}{|lllll|}
\hline Number & Protein Name & $\begin{array}{l}\text { Gene } \\
\text { Symbol }\end{array}$ & $\begin{array}{l}\text { Gene } \\
\text { ID }\end{array}$ & Degree \\
\hline 1 & serpin family E member 1 & SERPINE1 & 5054 & 15 \\
\hline 2 & vascular endothelial growth factor A & VEGFA & 7422 & 15 \\
\hline 3 & peroxisome proliferator activated receptor gamma & PPARG & 5468 & 15 \\
\hline 4 & nitric oxide synthase 3 & NOS3 & 4846 & 12 \\
\hline 5 & tumor protein p53 & TP53 & 7157 & 12 \\
\hline 6 & matrix metallopeptidase 9 & MMP9 & 4318 & 12 \\
\hline 7 & interleukin 10 & IL10 & 3586 & 12 \\
\hline 8 & adiponectin, C1Q and collagen domain containing & ADIPOQ & 9370 & 12 \\
\hline 9 & apolipoprotein B & APOB & 338 & 10 \\
\hline 10 & transforming growth factor beta 1 & TGFB1 & 7040 & 10 \\
\hline 11 & hypoxia inducible factor 1 alpha subunit & HIF1A & 3091 & 9 \\
\hline 12 & insulin like growth factor binding protein 3 & IGFBP3 & 3486 & 9 \\
\hline 13 & interleukin 4 & IL4 & 3565 & 8 \\
\hline 14 & sterol regulatory element binding transcription factor & SREBF1 & 6720 & 7 \\
\hline 15 & paraoxonase 1 & PON1 & 5444 & 6 \\
\hline 16 & cytochrome P450 family 3 subfamily A member 4 & CYP3A4 & 1576 & 4 \\
\hline 17 & glycogen synthase kinase 3 beta & GSK3B & 2932 & 4 \\
\hline 18 & matrix metallopeptidase 8 & MMP8 & 4317 & 4 \\
\hline
\end{tabular}

\section{Figures}




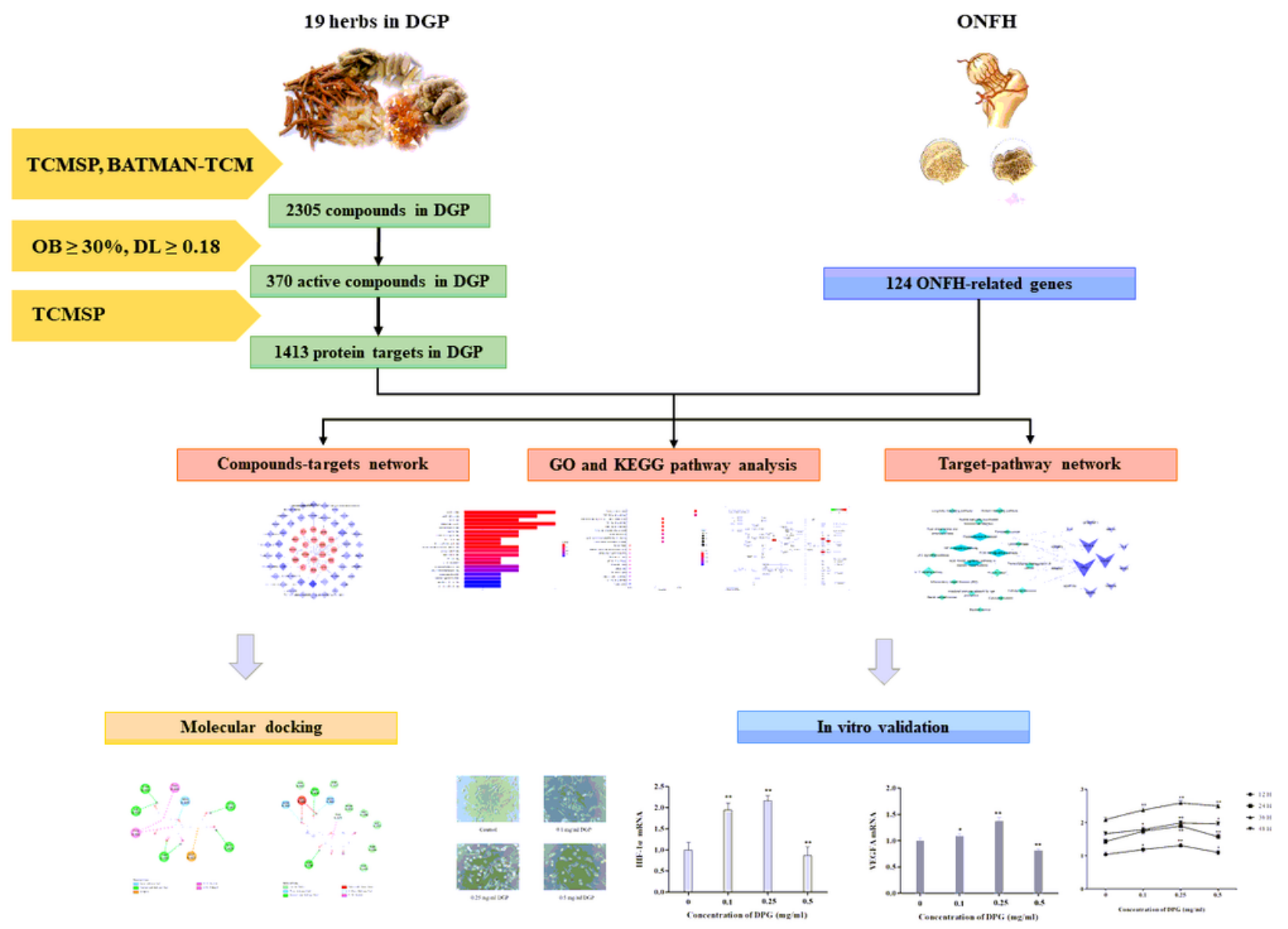

Figure 1

The technical strategy of the current study. 


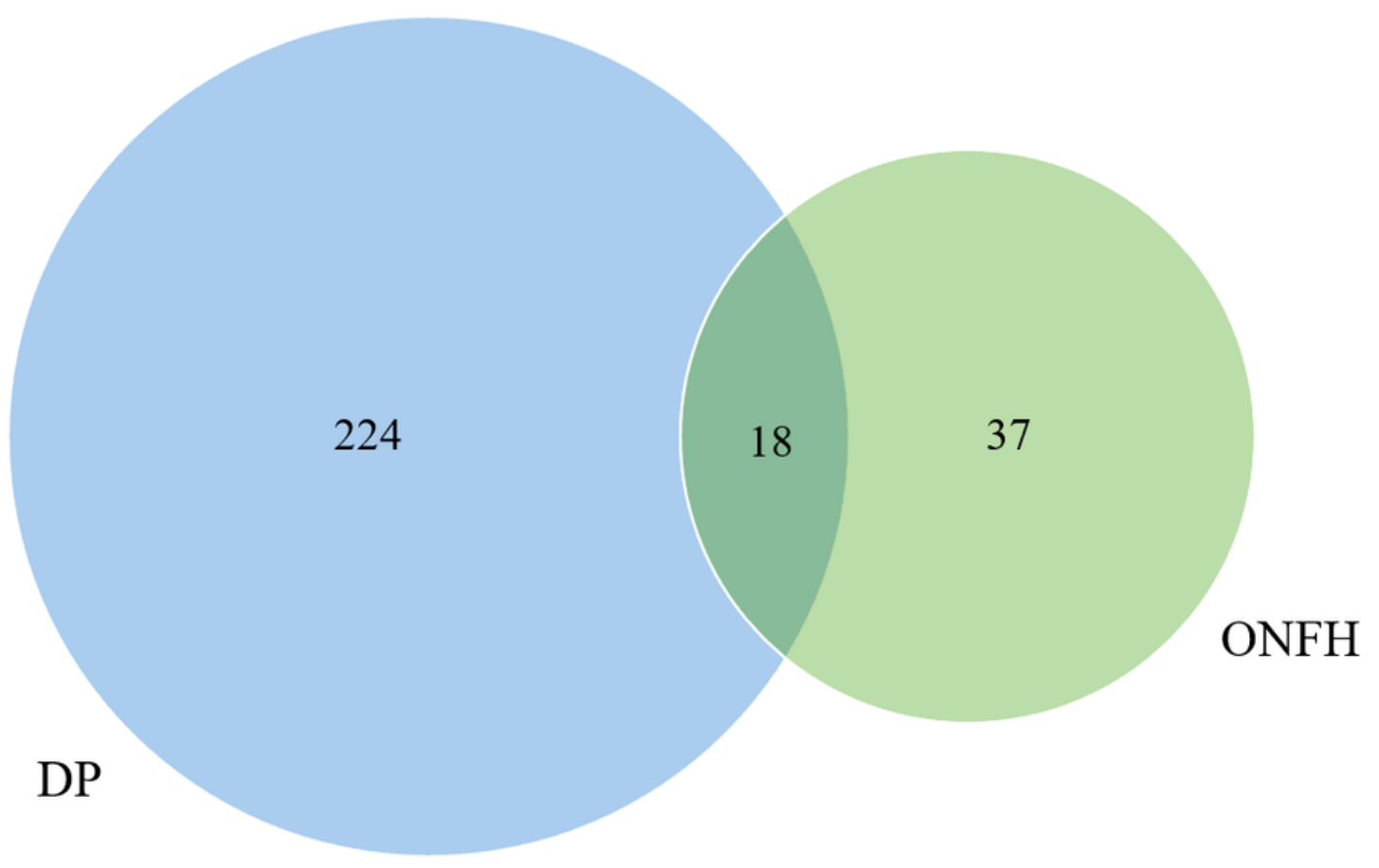

Figure 2

Venn diagram of drug targets and disease proteins. 


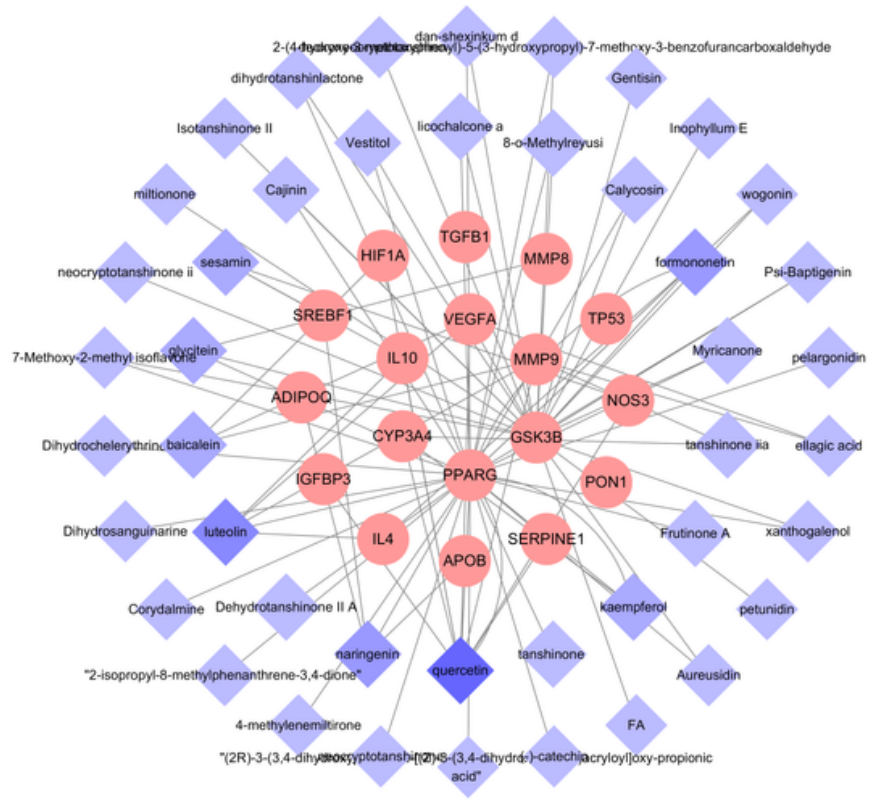

\section{Figure 3}

The compounds-target network for DGP on ONFH. The purple nodes represent candidate active compounds and the red nodes represent potential protein targets. The edges represent the interactions between them and shade of color is proportional to their degree.

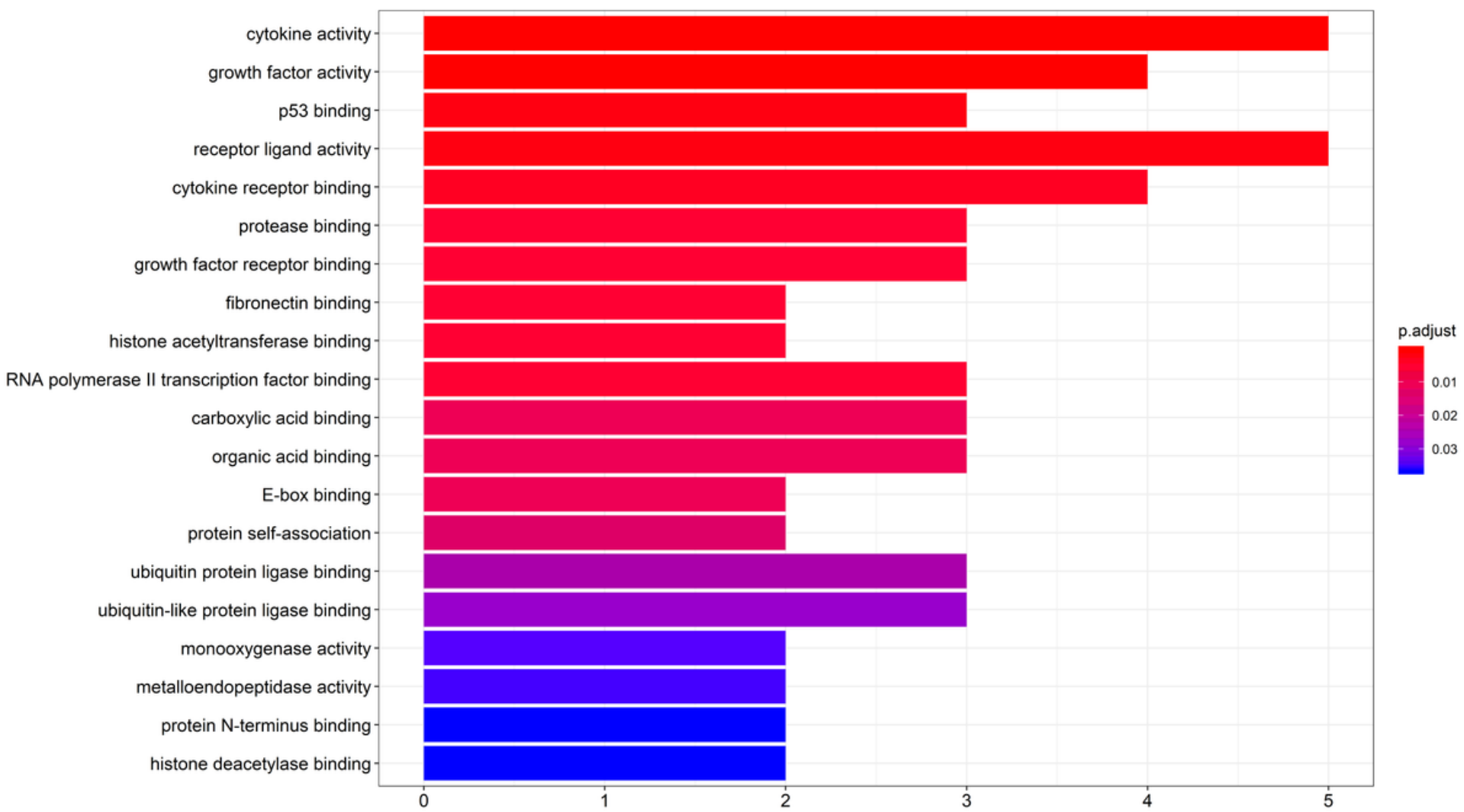




\section{Figure 4}

The 20 most significance of GO analysis of therapy target gene of DGP on ONFH.

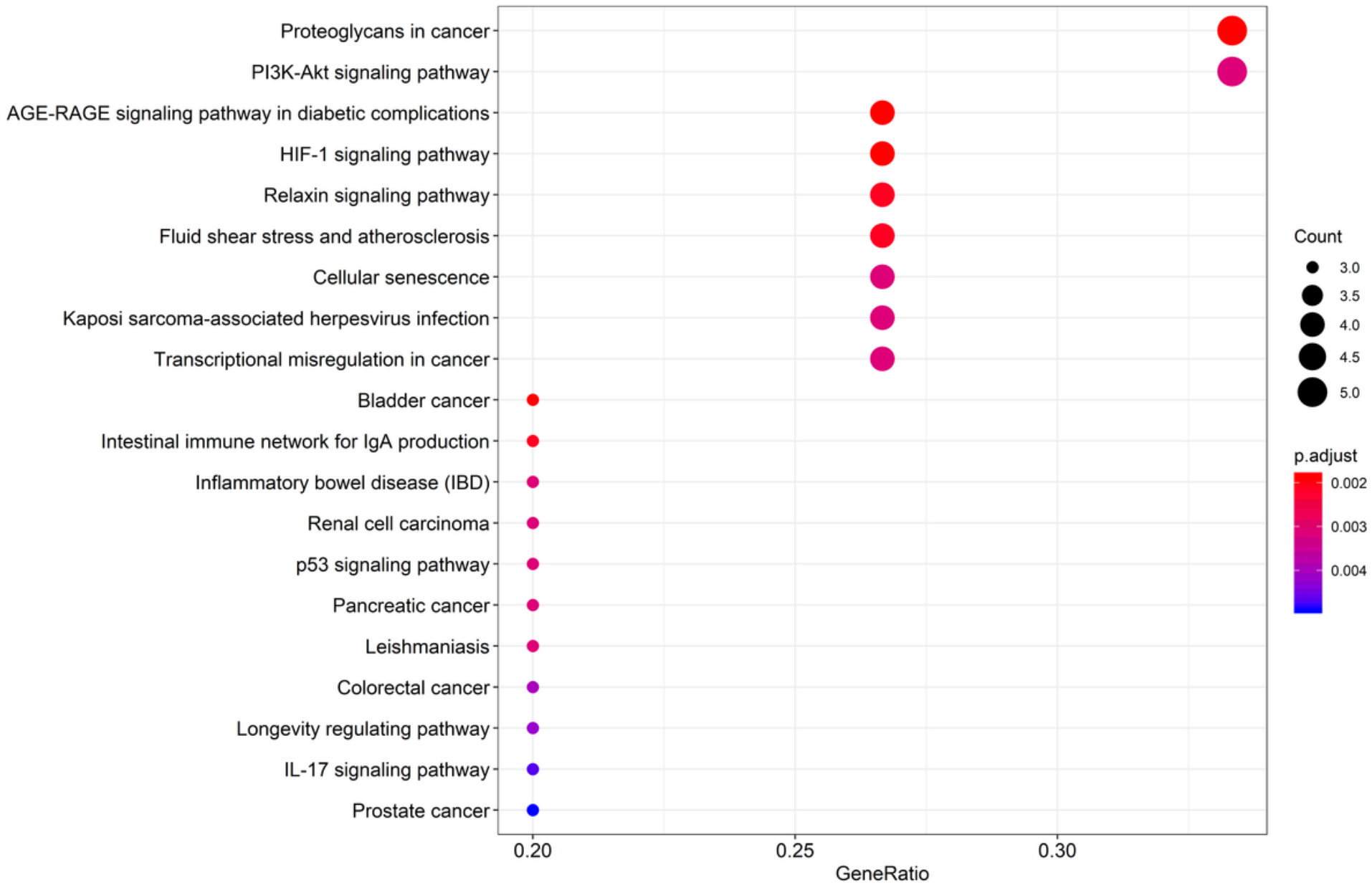

\section{Figure 5}

The 20 most significance of KEGG pathway enrich analysis $f$ therapy target gene of DGP on ONFH. 


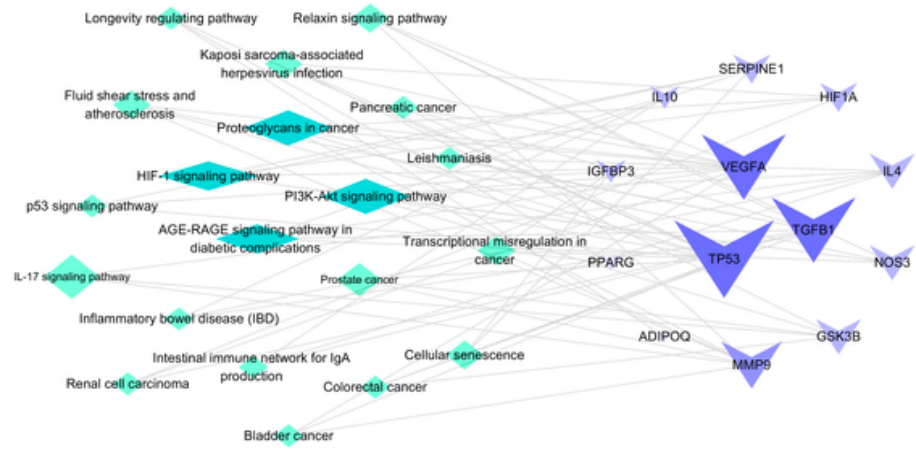

\section{Figure 6}

The target-pathway network for DGP on ONFH. The purple nodes represent targets and the green nodes represent pathways. The edges represent the interactions between them and node size is proportional to their degree. 


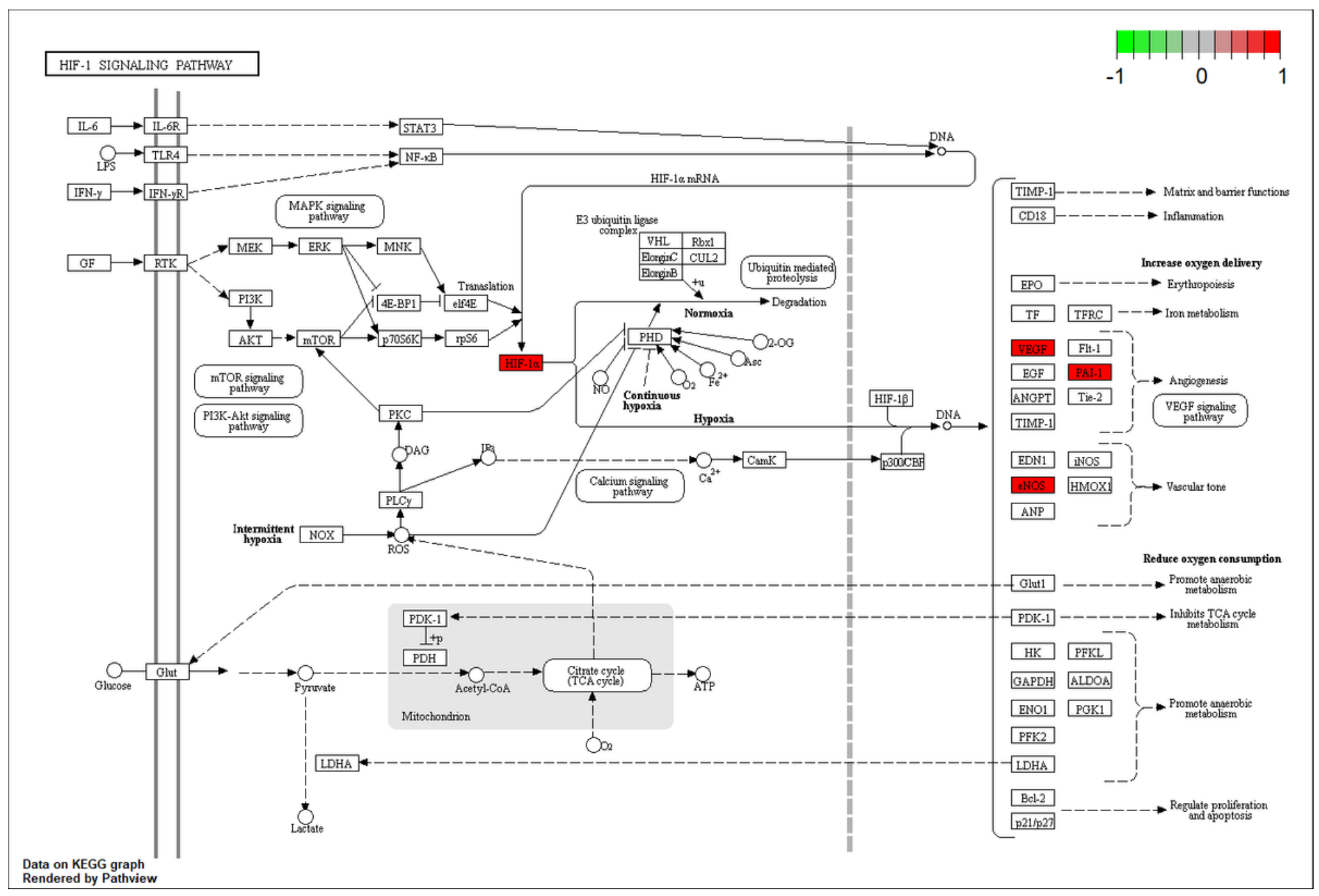

Figure 7

HIF-1 signaling pathway. 

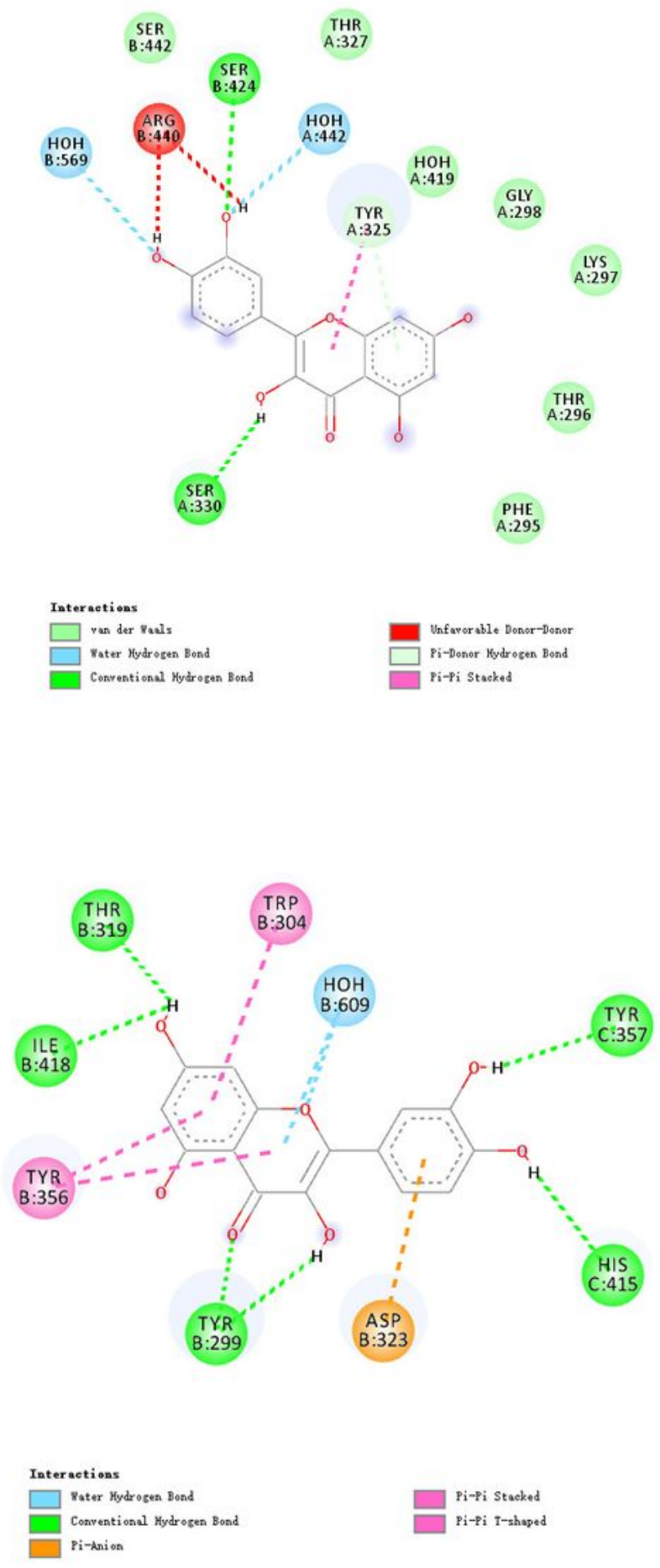

\section{Figure 8}

The docking patterns shown as 2D presentation of quercetin with (A) HIF-1a and (B) VEGFA. The red arrows refer to the amino acid residues that interact with quercetin in binding site of HIF-1a or VEGFA. 


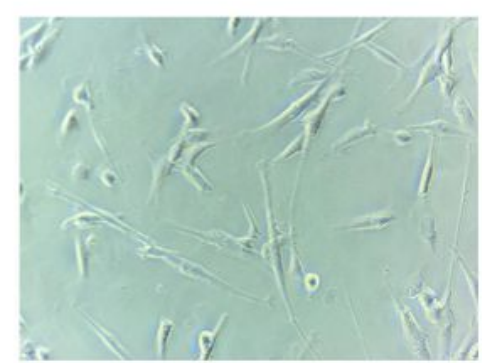

Control

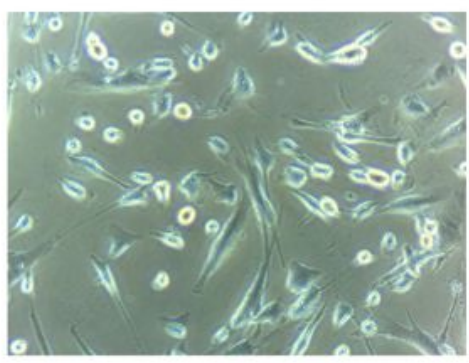

$0.25 \mathrm{mg} / \mathrm{ml}$ DGP

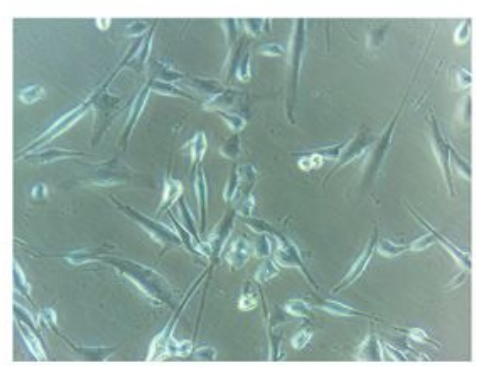

$0.1 \mathrm{mg} / \mathrm{ml} \mathrm{DGP}$

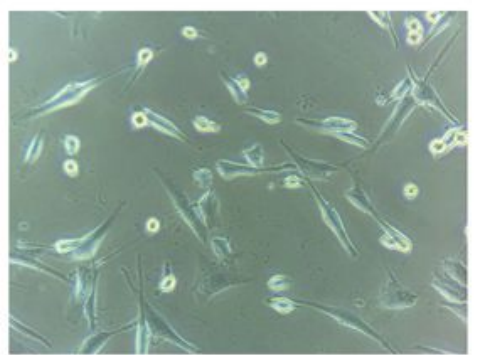

$0.5 \mathrm{mg} / \mathrm{ml}$ DGP

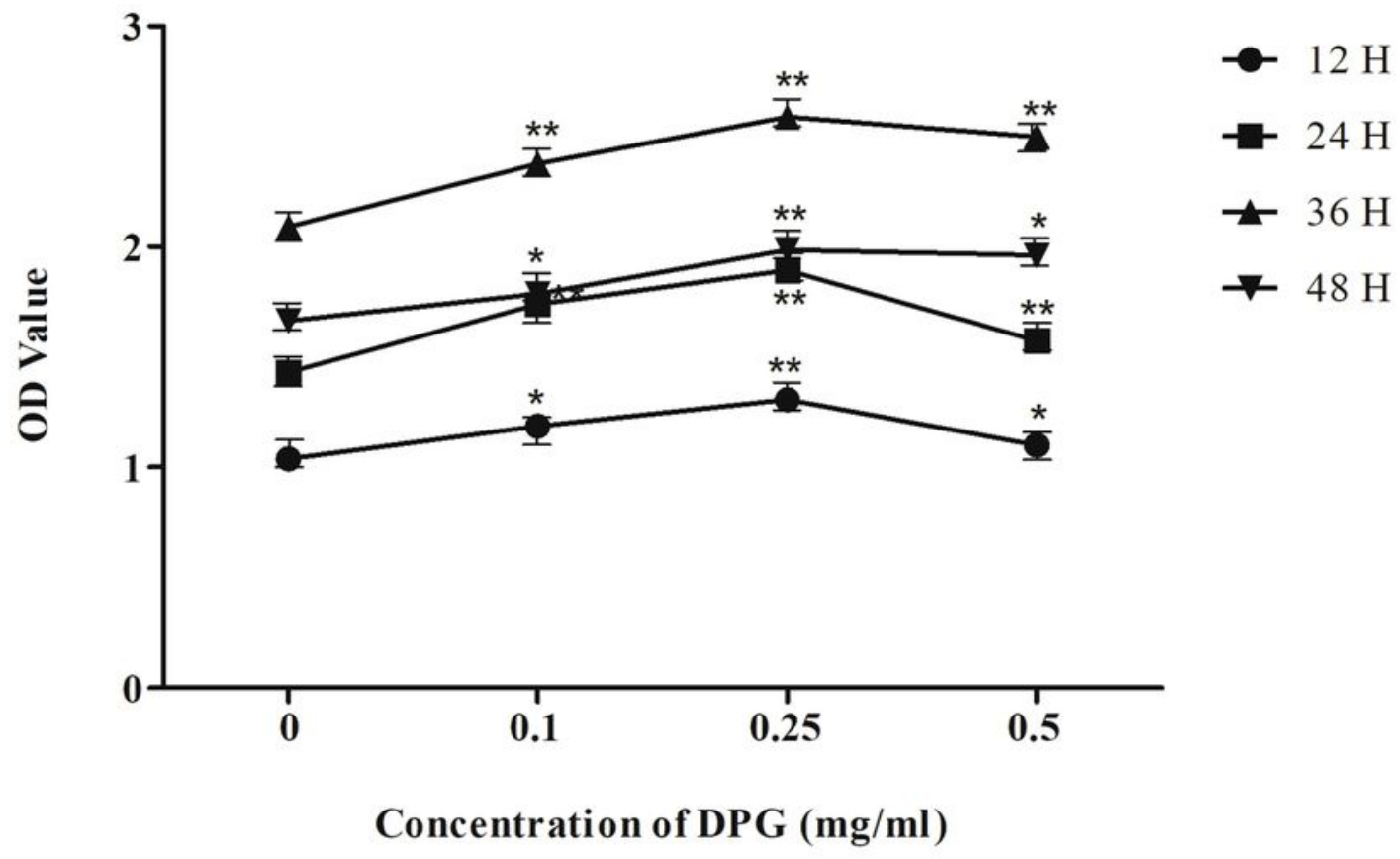

\section{Figure 9}

The effect of DGP on BMSCs viability. (A) BMSCs morphology under inverted microscope after DGP treating for $24 \mathrm{H}$. (B) BMSCs were treated with indicated concentration of DGP for 12, 24, $3648 \mathrm{H}$ and cell viability was tested using CCK-8 assay. The results are means of three independent replicates \pm S.D. * $\mathrm{p}$ values $<0.05$ and ${ }^{*}$ p values $<0.01$ vs control group were considered as statistically significant. 
A.

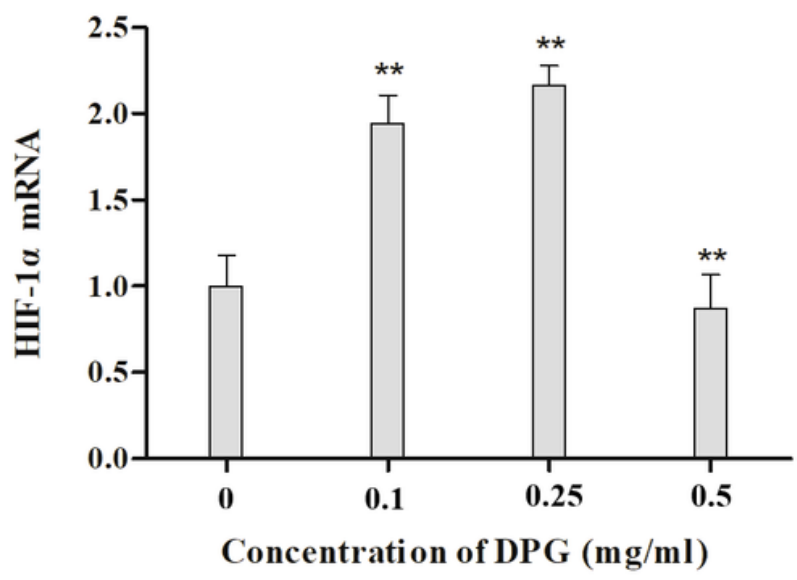

B.

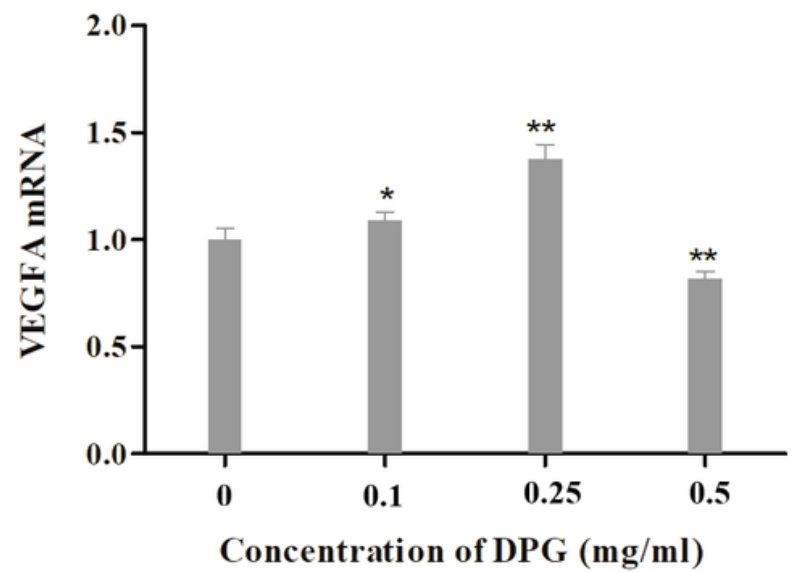

\section{Figure 10}

The mRNA expression of (A) HIF-1 $a$ and (B) VEGFA after indicated concentration of DGP treating for 24 $H$. The results are means of three independent replicates \pm S.D. ${ }^{*}$ p values $<0.05$ and ${ }^{* *} p$ values $<0.01$ vs control group were considered as statistically significant. 